\title{
Detection of Merkel Cell Carcinoma Polyomavirus in Mucosal Merkel Cell Carcinoma
}

Karen N. Wu, MD', Peter A. McCue, MD'1, Zi-Xuan Wang, PhD'1,2, and Agnieszka K. Witkiewicz, MD'1

${ }^{1}$ Department of Pathology, Anatomy and Cell Biology, ${ }^{2}$ Department of Surgery, Thomas Jefferson University, Philadelphia, PA.

\section{Case Report:}

A 61-year-old woman with a past medical history significant for hypertension, bipolar disorder, obstructive sleep apnea and chronic obstructive pulmonary disease presented with diffuse lymphadenopathy suspicious for lymphoma. A lymph node in her right groin was biopsied by fine needle aspiration and showed atypical cells, but was not diagnostic. An excisional biopsy of the $2 \times 2 \mathrm{~cm}$ lymph node demonstrated metastatic Merkel cell carcinoma (MCC). No skin lesions were detected. A CT scan revealed the presence of a large nasopharyngeal mass that following surgical removal was diagnosed as MCC. Treatment with etoposide and cisplatin and radiation were instituted. The patient went on to develop radiation-induced pneumonitis and cystitis, fungal sepsis, and deep vein thromboses. The patient was later transferred to a long-term acute care facility.

\section{Pathologic Findings:}

The microscopic examination of the nasopharyngeal tumor and the lymph node metastases showed a monotonous population of small- to intermediate-sized, round blue cells with vesicular nuclei and finely granular and dusty chromatin with multiple nucleoli. Immunohistochemistry revealed perinuclear staining in a dot-like pattern with cytokeratins AE1/AE3 and CK20. The cells were positive for c-kit and chromogranin and negative for LCA and S-100.This morphology and immunohistochemical profile were characteristic of MCC. MCC was recently shown to harbor a novel polyomavirus, Merkel Cell Polyomavirus (MCPyV), in the majority of cases (1). PCR analysis using the same primers as published (1) for T-antigen LT1 and LT3 was performed on formalin-fixed, paraffin-embedded (FFPE) tissue blocks from the patient's primary nasopharyngeal tumor and lymph node metastasis. MCPy $V$ was detected in the primary tumor and metastasis from 200ng of genomic DNA extracted from FFPE specimens.

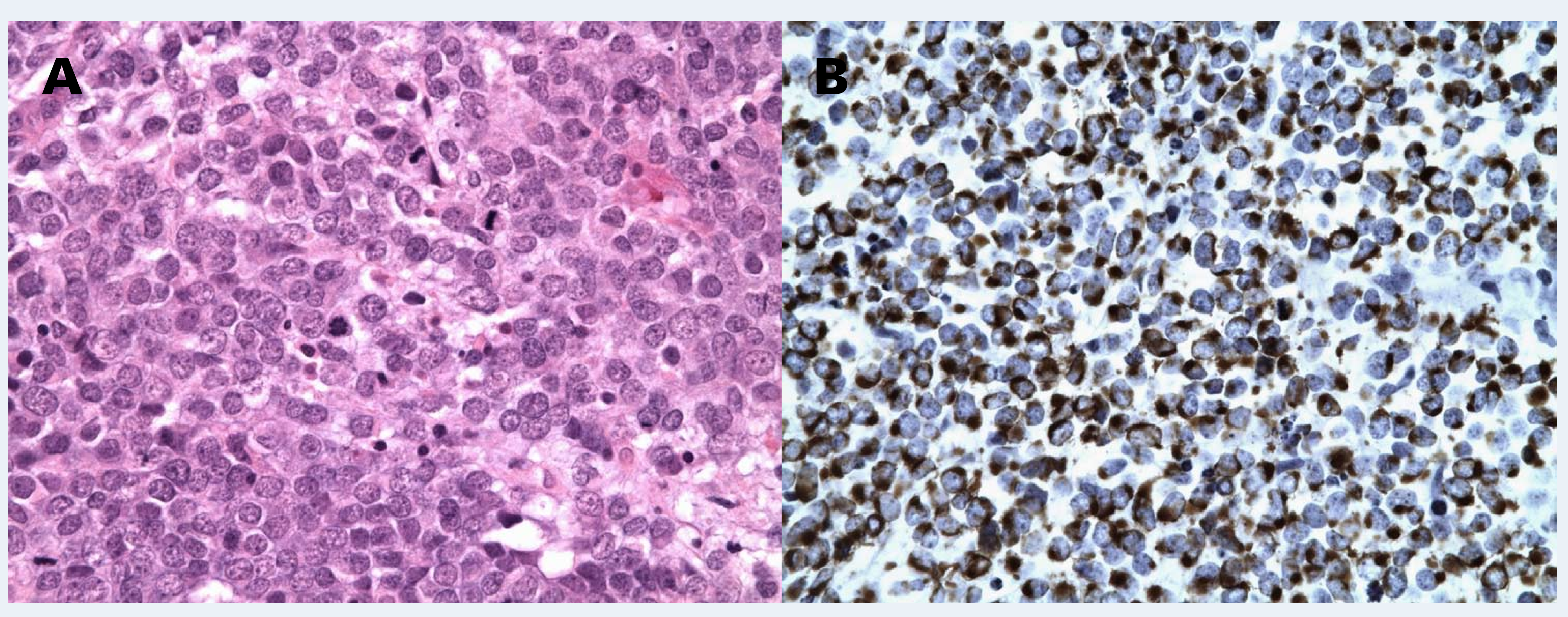

Figure 1. Primary nasopharyngeal MCC (A) Mucosal involvement of tumor H\&E (40x). (B) Perinuclear dot-like staining with CK20 (40x).

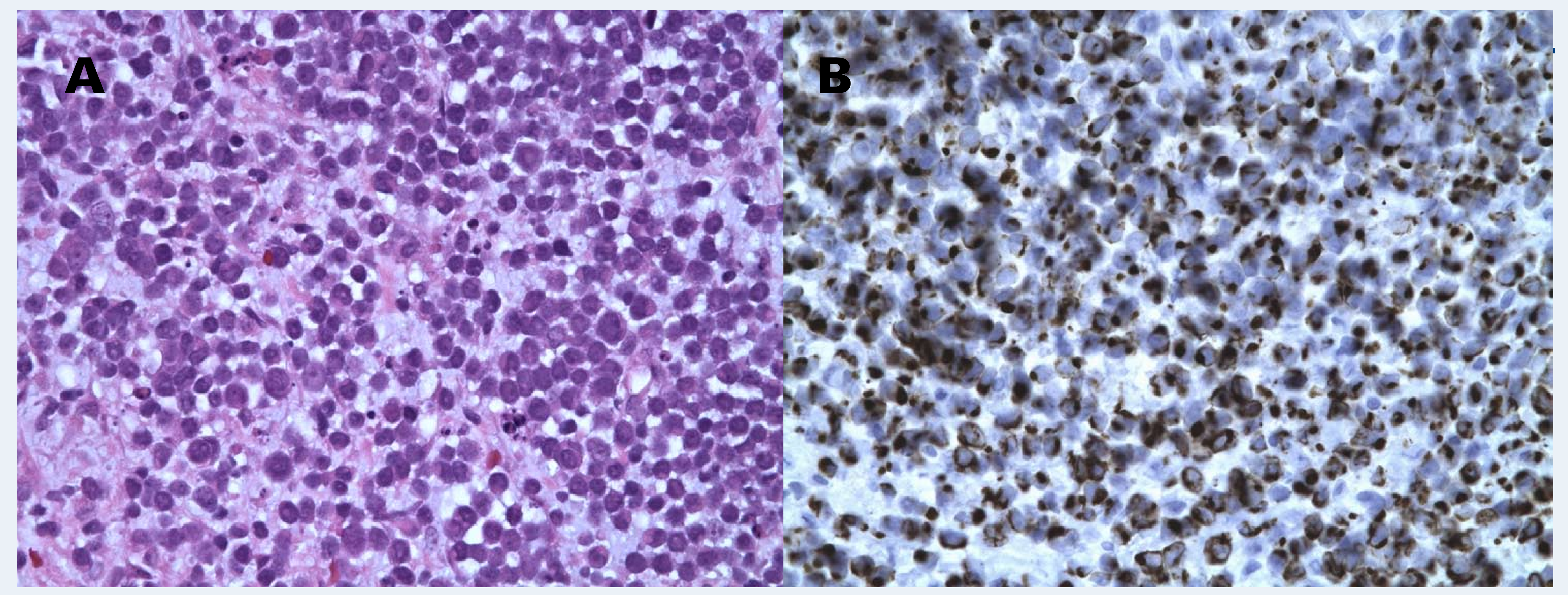

Figure 2. Metastatic MCC (A) Lymph node involvement of tumor cells, H\&E (40x). (B) Perinuclear dot-like staining with CK20 (40x).

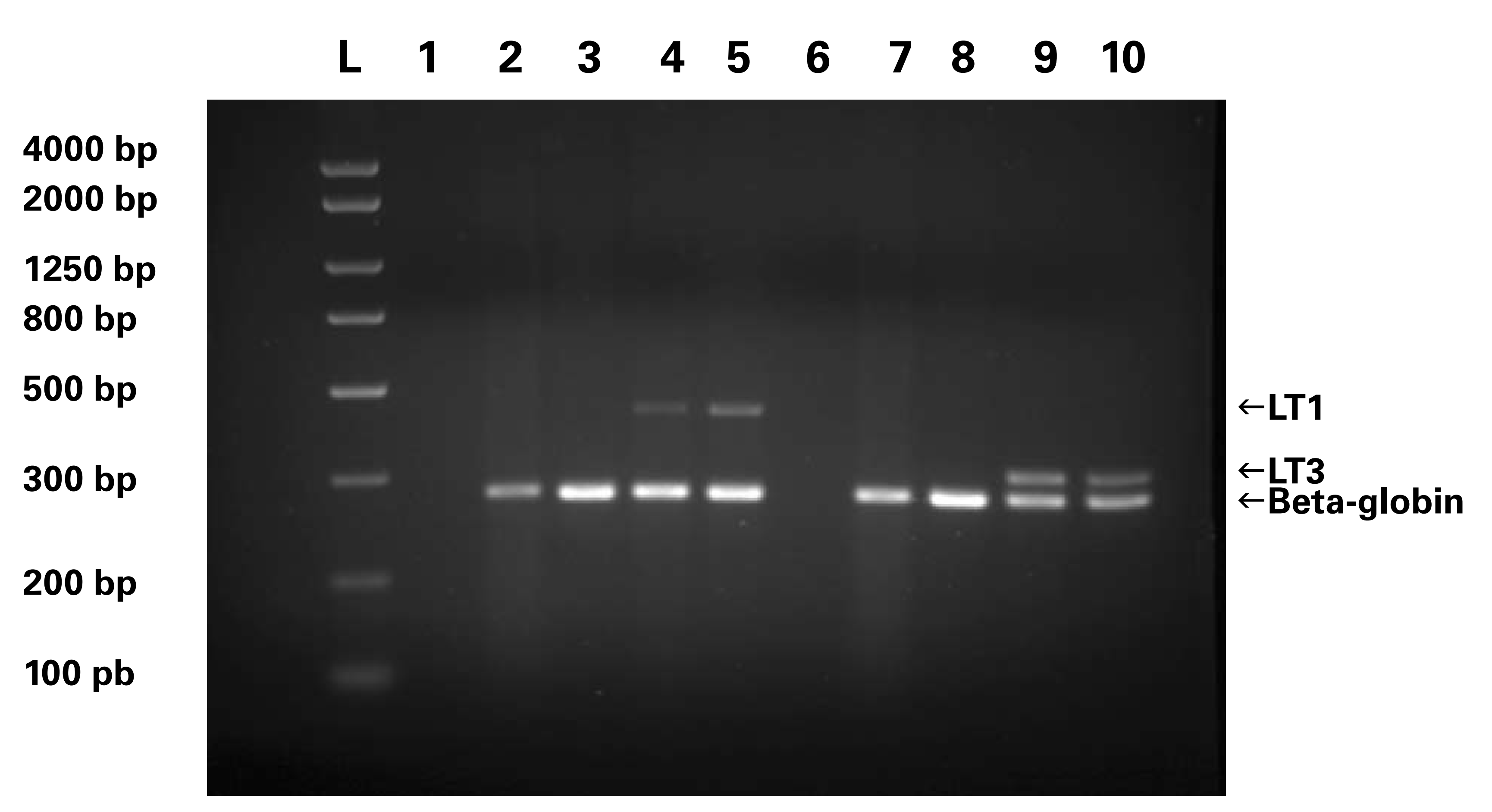

Figure 3. PCR result for detection of MCPyV from primary and metastatic MCC tumor specimens.

PCR was performed using the ApliTaq Gold (ABI), dNTP at $200 \mathrm{uM}$, primers at $200 \mathrm{nM}$ in $25 \mathrm{ul}$ volume. The cycling conditions were: $95^{\circ} \mathrm{C}$ for 10 min, 35 cycles consisting of $30 \mathrm{sec}$ at $94^{\circ} \mathrm{C}, 30 \mathrm{sec}$ at $55^{\circ} \mathrm{C}$, and $30 \mathrm{sec}$ at $72{ }^{\circ} \mathrm{C}$, then $72{ }^{\circ} \mathrm{C}$ for $7 \mathrm{~min}$. L, molecular weight ladder. Lanes 1 to 5 are multiplex PCR for LT1 viral gene (440 bp) and beta-globin housekeeping gene. 1, water control; 2 and 3, two colorectal cancer specimens as negative controls for the MCPyV; 4, primary nasopharyngeal MCC; 5 , metastatic MCC. Lanes 6 to 10 are multiplex PCR for LT3 (309 bp) and beta-globin genes; 6 , water control; 7 and 8 , two colorectal cancer specimens; 9, primary nasopharyngeal MCC; 10, metastatic MCC.

\section{Conclusion:}

Our report adds to the recent literature supporting a role for MCPyV in MCC. In addition, this is the first report to demonstrate presence of MCPyV in MCC arising in a mucosal site and in the metastatic foci.

Reference: 'Feng H, Shuda M, Chang Y, Moore P. Clonal integration of a polyomavirus in human Merkel cell carcinoma. 